\title{
SOCIO-EPIDEMIOLOGICAL EVALUATION OF TUBERCULOSIS AND IMPACT OF PHARMACEUTICAL CARE ON MEDICATION ADHERENCE AMONG TUBERCULOSIS PATIENTS
}

\author{
ARATHY THOMAS*, JOYAL JOY, ALAN KURIAN, SIVAKUMAR V
}

Department of Pharmacy Practice, PSG College of Pharmacy, Coimbatore, Tamil Nadu, India. Email: Rosethomas17.rt@gmail.com

Received: 11 June 2017, Revised and Accepted: 15 November 2017

ABSTRACT

Objectives: Tobjective of this study is to assess the knowledge and to monitor medication adherence among tuberculosis (TB) patients.

Methods: During the 6 months prospective interventional study, a data collection form was designed. The knowledge and adherence levels were assessed using quantified questionnaires. Patients were counseled using patient information leaflets and reassessed during follow-up.

Results: Results were analyzed using one-way ANOVA and Pearson's correlation.

Key findings: Among the 65 patients who completed 3 reviews during 6-month follow-up study, there was a statistically significant improvement in medication adherence score from baseline in correspondence with the increase in knowledge baseline to first follow-up revealed a mean increase in medication adherence level of $2.723 \pm 1.625(\mathrm{p}<0.05)$ in correspondence with a mean increase in knowledge level $24.8 \pm 5.04(\mathrm{p}<0.05)$ which is statistically significant. First follow-up to second follow-up showed a mean increase in medication adherence of $1.400 \pm 1.072$ (p<0.05) in correspondence with a mean increase in knowledge level $28.32 \pm 4.4(\mathrm{p}<0.05)$ which is statistically significant. Second to third follow-up showed a mean increase in medication adherence of $0.123 \pm 0.450(\mathrm{p}<0.05)$ in correspondence with a mean increase in knowledge level $34.18 \pm 2.74(\mathrm{p}<0.05)$ which is statistically significant.

Conclusion: The efforts to improve awareness on TB patients such as providing patient counseling and education could enhance adherence to therapies and thus could improve outcomes and quality of life.

Keywords: Knowledge, Medication adherence, Tuberculosis.

(C) 2018 The Authors. Published by Innovare Academic Sciences Pvt Ltd. This is an open access article under the CC BY license (http://creativecommons. org/licenses/by/4. 0/) DOI: http://dx.doi.org/10.22159/ajpcr.2018.v11i2.20503

\section{INTRODUCTION}

Tuberculosis (TB) is a highly contagious disease. It remains as a major cause of morbidity and mortality in these countries despite considerable declined prevalence in developed countries [1]

There are factors such as low socioeconomic status, substance abuse, and low education levels that will cause non-adherence from the start of treatment [2]. There are factors that are responsible for lack of knowledge, non-compliance with control program, inadequate diagnosis, and treatment. All the factors are rising due to population explosion, rise in a number of multidrug resistance, poor socioeconomic conditions, and lack of knowledge or education about TB and its treatment [3]. Still, compliance to treatment is a social stigma due to lack of knowledge of disease its treatment regimen [4]. Non-compliance to the administered medicines or multidrug anti-TB treatment regimen is common and important factor for failure of the initial therapy and relapse [5].

In the view of the above background, the study was conducted with the objective of assessing the social factors affecting the compliance to therapy and improving the patient adherence pattern by implementing pharmacist intervention in those areas of non-compliance.

\section{METHODS}

Study setting

The study is conducted at the inpatient department of respiratory medicine and general medicine. Patient diagnosed to have TB at PSG hospitals, which is 900 bed tertiary care hospital.

Study design

It was a prospective interventional study.
Study period

It was a prospective type of study initiated from April 2016 to September 2016.

Study criteria

The enrollment of patient was done as per the inclusion and exclusion criteria stated in the study protocol as follows:

The inclusion criteria are patients diagnosed and undergoing TB treatment, patients willing to participate in the study, and patients' age above 18 years of all the gender.

The exclusion criteria are patients with extrapulmonary TB, incomplete medical records, psychiatric illness, and pregnant and lactating women.

\section{Source of data}

Patient data collection form, hospital and medical records, and laboratory reports of TB patients are the sources of data.

\section{Ethical clearance}

The study was approved by PSG Institute of Medical Science and Research Institutional Ethics Committee and issued ethical clearance certificate with reference number 16/083 on $1^{\text {st }}$ April 2017.

\section{Informed consent}

Informed consent was obtained before enrollment of patients for the study.

Study materials

The following study materials were used for the study: 
- Patient data collection form

- Knowledge assessment interview

- Morisky adherence questionnaire

- Patient information leaflets(disease and drugs)

- Pictograms

- Patient informed consent.

Study procedure: After obtaining the approvals from the ethical committee, respiratory and general medicine department, the study was initiated at the respective departments by the enrollment of patients based on inclusion and exclusion criteria of the study.

The patients were explained about the study, and the consent form was given to sign in as the participant for the study. A suitably designed data collection form was prepared in consultation with the physician, clinical pharmacist, and also referring to standard textbooks and journals which include information of patient demographic details such as age gender, occupation, place of residence, education, type of TB, and duration of disease.

\section{Patient interview}

Baseline data regarding the patient knowledge and adherence level to treatment regimen were obtained by structured interview. During knowledge assessment, separate scores were given to the subjects based on their TB knowledge, attitude toward the disease, and disease prevention, and they were classified as excellent, average, and poor and provided information regarding the disease, signs and symptoms, its prevention, lifestyle modification, and treatment. Based on their Morisky adherence score, they were counseled and interviewed to understand the factors responsible for non-adherence to the treatment regimen. Patient education provided based on the adherence level. For low adherent patients, more number of tools was used for counseling. PILS prepared in English and vernacular languages separately for disease and drugs were distributed to TB patients as written educational materials.

Reassessment of the medication adherence and the knowledge once in every 2 months for 6-month duration was carried up during the follow-up.

\section{Statistical analysis}

Statistical analysis was performed using SPSS 16 version.

\section{RESULTS}

\section{Patient demographics}

Age

The age distribution is as follows: $22(24 \%)$ in the age group of 18 -35 years, $24(25 \%)$ in the age group of $36-45$ years, $18(17 \%)$ in the age group of 46-55 years, and $31(34 \%)$ in the age group of $>55$ years old. As per the results, maximum subjects (34\%) were in the age group $>55$ years old followed by $36-45$ years $(25 \%)$.

\section{Gender}

Among the enrolled TB patients, male patients were 72 (76\%) more compared to female $23(24 \%)$.

\section{Education background}

Among the participants of the study, 8 (12.30\%) of the patients were having formal education and were graduates; $5(7.69 \%)$ had a formal education upto higher secondary level; 21 (32.3\%) were having education up to junior high school level, and 31 (47.69\%) were educated at an elementary school level.

\section{Occupation}

$13(15 \%)$ patients were employed; 25 (25\%) patients were in the agriculture field; 13 (14\%) were businessman; 14 (15\%) were housewife; and $30(31 \%)$ of the patients belonged to other fields.

\section{Lifestyle habits \\ 28 (43.07\%) patients were alcoholic; 36 (55.38\%) were non-alcoholic; 37 (56.9\%) were smokers; $21(32.30 \%)$ were non-smokers; and $7(10.76 \%)$ were past smokers.}

\section{Place of residence}

It was found that $57.89 \%(n=55)$ of patients are from the small city and remaining $47.36 \%(\mathrm{n}=40)$ was from large city.

\section{Duration of disease}

Among the participants, 50 (76.9\%) of the patients were having TB for the past 1-5 months; $10(15.38 \%)$ of the patients were having TB for the past 6-10 months, and remaining $5(7.69 \%)$ were having TB for the past $\geq 11$ months.

\section{Type of TB}

Among the participants, $52(80 \%)$ of the patients suffered from PTB; $7(11 \%)$ of the patients were having multidrug-resistant TB; 6 (9\%) patients were having TB reactivation; and there was no patient with extensively drug-resistant TB detected.

\section{Medication adherence}

Medication adherence was classified as low adherence (3-8), medium adherence (0-2), and high adherence (0) based on Morisky 8 item questionnaire. In the baseline review, $72.3 \%(\mathrm{n}=47)$ had low adherence; $26.1 \%(\mathrm{n}=17)$ had medium adherence; and $1.5 \%(\mathrm{n}=1)$ were highly adhered to the treatment regimen.

\section{Comparison of medication adherence scores}

Medication adherence was assessed during baseline review, first review, second review, and final review. Comparing mean medication adherence between baseline (3.61 \pm 1.67$)$, first review $(2.72 \pm 1.62)$, second review $(1.40 \pm 1.07)$, and final review $(0.12 \pm 0.45)$ shows statistically significant improvement $(\mathrm{p}<0.05)$ in medication adherence (Table 1 and Fig 1).

Mean categorization of adherence barriers

The 3 topics of health education were asked to all TB patients, about knowledge toward disease and drugs (25), psychological aspects of the disease (9), and personal factors (7) and compared baseline mean of each category to the first, second, and final review. Each comparison showed a subsequent increase in the mean levels (Table 2 and Fig. 2).

Table 1: Comparison of mean adherence scores

\begin{tabular}{lll}
\hline Medication adherence & Mean \pm SD & p value \\
\hline Medication adherence baseline & $3.615 \pm 1.674$ & $\mathrm{p}<0.05$ \\
${\text { Medication adherence } 1^{\text {st }} \text { review }}^{\text {Medication adherence } 2^{\text {nd }} \text { review }}$ & $2.723 \pm 1.625$ & $\mathrm{p}<0.05$ \\
Medication adherence final review & $0.123 \pm 0.450$ & $\mathrm{p}<0.05$ \\
\hline
\end{tabular}

SD: Standard deviation

Table 2: Categorization of adherence barriers and mean values

\begin{tabular}{llll}
\hline Barriers & Baseline & $\mathbf{1}^{\text {st }}$ review & Final review \\
\hline Knowledge & $11.22 \pm 3.20$ & $13.38 \pm 2.83$ & $15.29 \pm 2.50$ \\
Psychological & $5.63 \pm 1.58$ & $6.71 \pm 1.221$ & $7.40 \pm 0.997$ \\
Personal & $4.20 \pm 1.121$ & $4.71 \pm 0.996$ & $5.63 \pm 0.911$ \\
\hline
\end{tabular}


Mean knowledge assessment

Knowledge was assessed during baseline review, first review, second review, and final review. Comparing mean knowledge between baseline (21), first review (24.74), second review (28.34), and final review (31.72) shows statistically significant improvement $(\mathrm{p}<0.05)$ in knowledge (Table 3 and Fig. 3).

\section{Correlation analysis}

The statistical analysis used for the purpose was Pearson's correlation, $\mathrm{p}<0.01$ for finding association between two groups. In this study, we analyzed the correlation between medication adherence and various socio-epidemiological factors such as gender, age, education, place of residence, and occupation (Table 4).

The value of significance ( 2 sided) for correlation should be $<0.01$ (i.e., $\mathrm{p}<0.01$ ) so that there is a significant association between two groups.

\section{DISCUSSION}

A prospective interventional study was carried out for 6 months in a tertiary care hospital to evaluate two main objectives, i.e., to improve the medication adherence, patient knowledge using a standard questionnaire and patient information leaflet. Through literature survey, we found that the major determinants for the improvement of medication adherence and knowledge are providing continuous education and patient counselling.

The majority of the patient population was under the age group of above 55 years. Among the 95 patients, 76\% were males and remaining 24\% were females. Kgupta et al., in a socio- epidemiological cross-sectional survey in Jammu and Kashmir (2002), said that among the various socio-epidemiological variables age and sex being the non-modifiable. In this study, a majority of the cases were in the male population (16 cases), and 10 of 25 were in the age group of 15-45 years [6]. In a study conducted by NTI it was found out that the prevalence of the TB is more in the young and the middle age group [7].

In this study, there is an association between age and medication adherence as the age increases, medication adherence or compliance decreases. Association between gender and medication adherence is statistically insignificant. These findings are inconsistent with the study conducted by $\mathrm{N}$ panditetal. The sociodemographic factors (age and sex) were not associated significantly with adherence [5,8]. Gad et al. conducted a study in Alexandria in 1997 also conveyed the same fact [9]. Our study reveals low educational levels as one of the major reasons for the low adherence of patients. The study revealed that the compliance of DOT was significantly high among those who have good knowledge about the various aspect of disease; similar dissociations have been documented by other author in their study [2,5,9-14].

In our study, a significant association was found between educational level and adherence. These findings are consistent with the study conducted by Choi et al., low educational levels were associated with poor adherence [9].
Among the social variables, studied type of residence found to be statistically insignificant in relation to patient's compliance. In contrast to this, Gupta et al. told that overcrowding was found to be statistically significant in relation to the outcome of TB [6]. A study conducted by Drucker et al. at New York in 1993 confirmed that the association of overcrowding with poor adherence of TB [15].

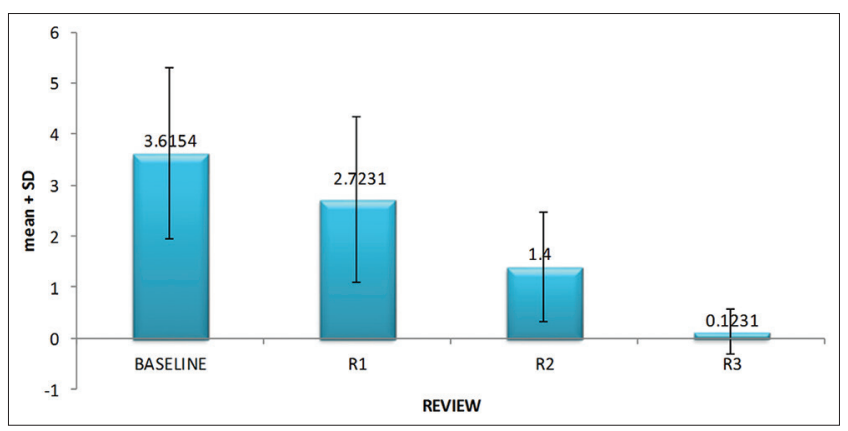

Fig. 1: Comparison of mean adherence scores



Fig. 2: Comparison of mean adherence barriers

Table 3: Comparison of mean knowledge assessment

\begin{tabular}{lll}
\hline Knowledge assessment & Mean & p value \\
\hline Knowledge assessment baseline & $21 \pm 3.90$ & 0.05 \\
Knowledge assessment $1^{\text {st }}$ review & $24.74 \pm 3.36$ & 0.05 \\
Knowledge assessment $2^{\text {nd }}$ review & $28.34 \pm 3.00$ & 0.05 \\
Knowledge assessment $3^{\text {rd }}$ review & $31.72 \pm 3.049$ & 0.05 \\
\hline
\end{tabular}

Table 4: Correlation analysis of socio-epidemiological factors

\begin{tabular}{lll}
\hline Factor & Correlation coefficient & p value \\
\hline Age & 0.020 & 0.05 \\
Gender & 0.141 & 0.05 \\
Education & 0.000 & 0.01 \\
Occupation & 0.784 & 0.05 \\
Place of residence & 0.623 & 0.05 \\
\hline
\end{tabular}

Table 5: Self-reported reason to stop the medications

\begin{tabular}{ll}
\hline Reasons & Number of patients n=95 (\%) \\
\hline Lack of information about disease & $35(36.8)$ \\
Fear of side effects & $25(26.3)$ \\
High cost of medications and diagnosis & $30(31.5)$ \\
Lack of assess to hospital/drug store & $38(40)$ \\
Fear of being dependent on treatment & $20(21)$ \\
Stopping medicines once the symptoms are over & $60(63.1)$ \\
Believed medications were not effective to take the dose & $15(15.7)$ \\
Lack of family support & $23(24.2)$ \\
Long duration of treatment & $47(49.4)$ \\
Absorbed in daily work and forget to takecupation related & $50(52.6)$ \\
Forgetfulness & $49(51.7)$ \\
\hline
\end{tabular}


Table 6: Correlations between knowledge and medication adherence

\begin{tabular}{lll}
\hline Review & Correlation coefficient & p value \\
\hline Baseline & -0.147 & 0.05 \\
First review & -0.210 & 0.05 \\
Second review & -0.419 & 0.05 \\
Final review & -0.484 & 0.05 \\
\hline
\end{tabular}

The main risk factors for non-compliance were studied and they were forgetfulness, high cost of treatment, long duration, lack of access to medical store, fear of side effects, confusion over the schedule, lack of information about how to take the drug absorbed in work and forgot to take the medicine, lack of family support, and fear of being dependent on treatment (Table 5). A similar observation has been documented by other authors in their study $[5,11,16]$.

The problems were identified based on the self-reported reasons to stop medications that lead to non-adherence of TB patients. According to our study, most of the patients believed that the symptoms were over and they stopped taking medicine (63.1\%) followed by people who compliant or had problem of taking medicines when going for daily work (52.6\%). The least reported self-medication problem was, avoiding the dose believing that medication was not effective and decided not to take the dose (15.7\%). Bhardwaj et al. said that according to their study forgetfulness (58.97\%) was the self-reported medication problem and least reported was high cost of medications (30.77\%) and lack of information to take drug (30.77\%) [16].

\section{CONCLUSION}

Lack of knowledge regarding the disease is the most important factor that leads to non-compliance. Non-compliance requires more prolong and expensive treatment that is less likely to be successful than the drug-susceptible TB.

The impact of structured education showed significant improvement on knowledge about disease and medication adherence in TB patients. This will improve the awareness of patients on TB. The noncompliance associated factor identified was patient's poor knowledge regarding disease condition and therapy. Education provided by clinical pharmacists resulted in a considerable increase in the medication adherence and knowledge at the end of the study (Table 6 and Fig. 4). Thus, our study concludes that efforts to improve awareness on TB patients such as providing patient counseling and continuous education could enhance adherence to therapies and thus could improve clinical outcomes and quality of life of TB patient.

\section{ACKNOWLEDGMENT}

The authors thank PSG College of Pharmacy and PSG Hospitals for providing facilities and support to carry out this research.

\section{AUTHORS CONTRIBUTION}

AT and JJ developed the protocol and conceptualized the study. AT and AK analysed the data, with later analysis supported by all authors. V.K wrote the initial draft. JJ and AK edited the manuscript; and all authors contributed to the final draft.

\section{CONFLICT OF INTEREST}

No conflicts of interest.

\section{REFERENCES}

1. Olakunale OS, Olanrewaju O, Olalekan AW, Bello AO, Akinleye C, Oluwatoyin OA. Knowledge of tuberculosis management using directly observed treatment short course therapy among final year

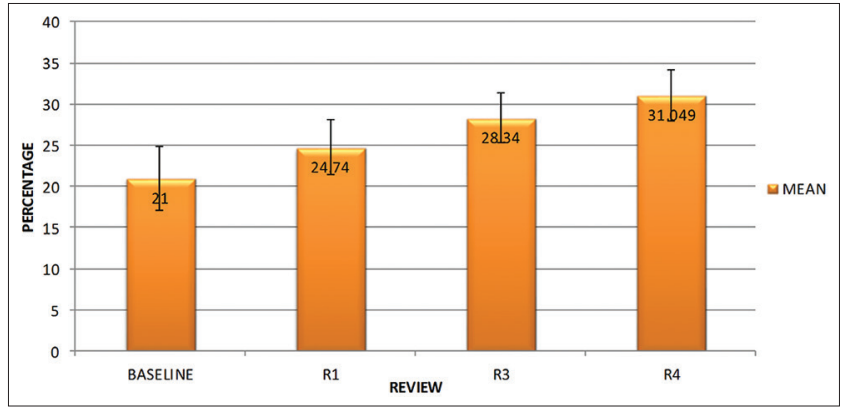

Fig. 3: Comparison of knowledge assessment



Fig. 4: Knowledge-adherences correlation

medical students in south western Nigeria. Afr Med J 2014;5:1-5.

2. Choi H, Chung H, Muntaner C, Lee M, Kim Y, Barry CE, et al. The impact of social conditions on patient adherence to pulmonary tuberculosis treatment. Int J Tuberc Lung Dis 2016;20:948-54.

3. Rehman F, Rao S, Hasan A, Parmehwar H, Parameshwar H, Raoof MA, et al. An assessment of patient's perception on tuberculosis, prevention and control in a teaching hospital. Adv Pharmacoepidemiol Drug Saf 2016;5:201.

4. Sukumaran P, Venugopal KP, Manjooran RS. A study of compliance with DOTS. Indian J Tuberc 2002;49:205-8.

5. Pandit N, Choudhary SK. A study of treatment compliance in directly observed therapy for tuberculosis. Indian J Community Med 2006;31:241-3.

6. Gupta RK, Gupta A, Jamwal DS, Suri SP. A socio-epidemiological study of tuberculosis in a rural area. JK Science 2002;4:20-2.

7. Tuberculosis in a rural population of South India: A five-year epidemiological study. Bull World Health Organ 1974;51:473-88.

8. Sumartojo E. When tuberculosis treatment fails: A social behaviour account of patient adherence. Am Rev Respir Dis 1993;147:1311-8.

9. Gad A. Compliance with anti-tuberculosis drugs among tuberculosis patients in Alexandria, Egypt. PLosMed 1997;3:244-50.

10. Solliman MA, Hassali MA, Haddad MA, Hadida MM, Saleem F, Atif M, et al. Assessment of knowledge towards tuberculosis among the general population in North East Libya. J Appl Pharm Sci 2012;2:24-30.

11. Stewart MA. What is successful doctor patient interview? A study interactions and outcomes. Soc Sci Med 1984;19:167-75.

12. Tekle B, Mariam DH, Ali A. Defaulting from DOTS and its determinants in three districts of Arsi zone in Ethiopia. Int J Tuberc Lung Dis 2002;6:573-9.

13. Boyle SJ, Power JJ, Ibrahim MY, Watson JP. Factors affecting patient compliance with ant tuberculosis chemotherapy using the directly observed treatment, short course strategy (DOTS). Int J Tuberc Lung Dis 2002;6:307-12.

14. Thomas C. A literature review of the problems of delayed presentation for treatment and non-completion of treatment for tuberculosis in less developed countries and ways of addressing these problems using particular implementations of the DOTS strategy. J Manag Med 2002;16:371-400.

15. Drucker E, Elcabes P, Bosworth W. Childhood tuberculosis in Bronx (New York). Lancet 1994;343:1482.

16. Bhardwaj A, Kumar R, Dabas V, Alam N. Assessment and enhancing adherence to treatment regimen in tuberculosis outpatients. Int J Pharm Pharma Sci 2012;4:517-22 